DOI: $10.6060 / \mathrm{rcj} .2021653 .1$

УДК: 539.1.044: 535.683

\title{
ОТРИЦАТЕЛЬНЫЙ ФОТОХРОМИЗМ: ПОСЛЕДНИЕ ДОСТИЖЕНИЯ
}

\section{В.А. Барачевский}

Центр фотохимии Федерального научно-исследовательского центра «Кристаллография и фотоника» Российской академии наук, ул. Новаторов, д. 7а, к.1, Москва, Российская Федерация, 119421

Межведомственный центр аналитических исследований в области физики, химии и биологии при Президиуме Российской академии наук, ул. Профсоюзная ул., д. 65, стр.6, Москва, Российская Федерация, 117997

E-mail: barachevsky@mail.ru

Представлен анализ последних достижений в исследовании явления отрицательного фотохромизма органических соединений из классов спиропиранов, Стенхаус и нитрил-содержацих соединений, бииимидазолильных радикальныbх комплексов, дигидропиранов, цианиновых красителей, азуленов и гидразонов. Установлены зависимости спектрально-кинетических свойств от их структуры и природы заместителей. Рассмотрены возможности применения фотохромных систем на их основе в создании покрытий с фотоадаптивными свойствами и в биомедицинких технологиях.

Ключевые слова: отрицательный фотохромизм, фотохромные соединения, спектроскопия

\section{NEGATIVE PHOTOCHROMISM: RECENT ADVANCES}

\author{
V.A. Barachevsky
}

Photochemistry Centre of the Federal Scientific Research Centre "Crystallography and Photonics" of the Russian Academy of Sciences. Street Novatorov, 7a, bld. 1, Moscow, Russian Federation, 119421

Interdepartmental Center of Analytical Research in the field of physics, chemistry and biology at the Presidium of the Russian Academy of Sciences. Street Profsoyuznaya 65, bld. 6, Moscow, Russian Federation, 117997

E-mail: barachevsky@mail.ru

An analysis of the latest advances in the study of the phenomenon of negative photochromism of organic compounds from the classes of spiropyrans, Stenhouse and nitrile-containing compounds, biimidazolyl radical complexes, dihydropyrans, cyanine dyes, azulenes and hydrazones is presented. The dependences of spectral-kinetic properties on their structure and the nature of substituents have been established. The possibilities of application of photochromic systems based on them in the development of coatings with photoadaptive properties and in biomedical technologies are considered.

Key words: negative photochromism, photochromic compounds, spectroscopy

Для цитирования:

Барачевский В.А. Отрицательный фотохромизм: последние достижения. Рос. хим. ж. (Ж. Рос. хим. об-ва). 2021. T. LXV. № 3. C. 6-18

For citation:

Barachevsky V.A. Negative photochromism: recent advances. Ros. Khim. Zh. 2021. V. 65. N 3. P. 6-18

\section{ВВЕДЕНИЕ}

Фотохромизм органических соединений остается одним из инновационных направлений развития современной фотохимии [1]. Основные усилия исследователей, работающих в области фотохромизма направлены на синтез новых фотохромных соединений, систем и материалов на их 
основе, проявляющих положительный фотохромизм, т.е. обратимое фотоокрашивание веществ. Одним из практически значимых результатом в этом направлении является создание фотохромных пластиковых линз офтальмологического назначения [2]. Разработаны основы создания фотохромных регистрирующих сред для 3D оптической памяти [3].

Значительно меньшее внимание уделяется исследованию явления отрицательного фотохромизма, когда соединения, системы и материалы на их основе обратимо теряют окраску под действием видимого излучения. Материалы с отрицательным фотохромизмом не только расширяют функциональные возможности фотохромизма, но и могут обладать высоким ресурсом работы, поскольку, в отличие от материалов с положительным фотохромизмом, для осуществления фотохромных превращений используется не высокоэнергетическое УФ, а низкоэнергетическое видимое излучение.

Анализ результатов исследований, выполненных ранее в области отрицательного фотохромизма органических соединений $[4,5]$, показывает, что это направление еще не получило должного развития.

Настоящий обзор является продолжением ранее выполненного анализа результатов исследований, в котором отражены достижения в области отрицательного фотохромизма за последние 5 лет.

\section{ФОТОХРОМНЫЕ СИСТЕМЫ С ОТРИЦАТЕЛЬНЫМ ФОТОХРОМИЗМОМ}

\section{Спиросоединения}

К числу наиболее исследуемых фотохромных соединений с отрицательным фотохромизмом относятся спиропираны, испытывающие обратимые фотопревращения между формами А и В согласно схеме 1 [1].

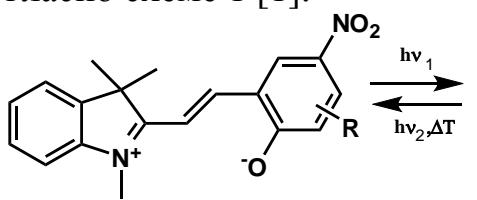

A

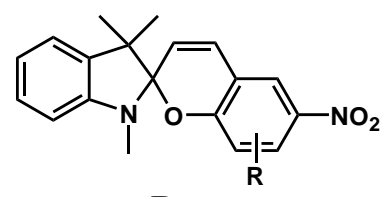

B
Схема 1

Систематическое спектрально-кинетическое исследование процессов комплексообразования ряда нитрозамещенных индолиновых спиропиранов СП 1-СП 3 показало, что все они при взаимодействии с ионами металлов образуют окрашенные комплексы, которые проявляют негативный фотохромизм как в растворах (рис. 1), так и в полимерных связующих [6].<smiles>[R]c1cc([N+](=O)[O-])cc2c1OC1(C=C2)N([14CH])c2ccccc2C1(C)C</smiles>

$\mathrm{C \Pi}$ 1: $\mathrm{R}_{1}=\mathrm{CH}_{3} ; \mathrm{R}_{2}=\mathrm{OCH}_{3}$

CП 2: $\mathrm{R}_{1}=\mathrm{CH}_{3} \mathrm{CH}_{2} \mathrm{OH} ; \mathrm{R}_{2}=\mathrm{OCH}_{3}$

CП 3: $\mathrm{R}_{1}=\mathrm{CH}_{3} ; \mathrm{R}_{2}=\mathrm{OCH}\left(\mathrm{CH}_{3}\right)_{2}$

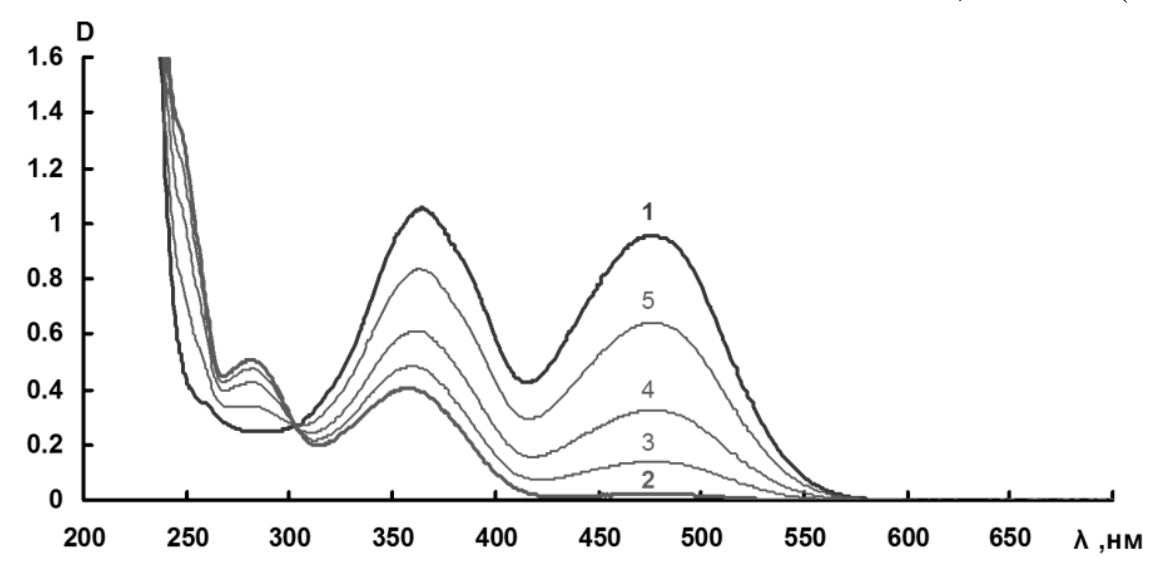

Рис. 1. Спектры поглощения СП 1 в ацетонитриле $\left(\mathrm{C}=2,10^{-4} \mathrm{M}\right)$ в присутствии в растворе катиона $\mathrm{Tb}^{3+}$ до $(1)$, после облучения видимым светом (2) и в процессе темновой релаксации (3-5).

[CП] : $[\mathrm{Me}]=1: 100$

Фотоиндуцированное динамическое изменение окраски комплексов, проявляющих отрицательный фотохромизм, определяется не только изменением поглощения, но и интенсивности люминесценции [7].
Окраска комплексов молекул и положение полос поглощения раствора мероцианиновой формы комплексов молекул спиропиранов с ионами металлов зависит от природы металла и структуры фотохромного соединения [6]. Максимум этой полосы 
поглощения гипсохромно сдвигается до 100 нм относительно аналогичной полосы поглощения раствора, не содержащего ионы металлов, с увеличением сродства ионов к электрону.

В случае фотохромных полимерных систем спектральные и релаксационные свойства комплексов зависят также от природы полимерного связующего. В отличие от растворов обнаружены спектральные изменения в процессе хранении полимерных слоев в темноте, обусловленные образованием протонированных форм мероцианиновой формы вместо первоначально возникающих металлокомплексов. Эффективность протонирования мероцианиновой формы зависит от содержания гидроксильных групп в составе полимерного связующего и стабильности металлокомплексов.

Отрицательный фотохромизм проявляют растворы комплексов 8-(1,3-бензоксазол-2-ил) замещенных спиропиранов СП 4-СП 7 с ионами металлов [8]. При этом в отличие от ионов $\mathrm{Co}^{2+}, \mathrm{Ni}^{2+}$, $\mathrm{Cu}^{2+}$ ионы $\mathrm{Mn}^{2+}, \mathrm{Cd}^{2+}$ и $\mathrm{Zn}^{2+}$ образуют люминесцирующие комплексы.

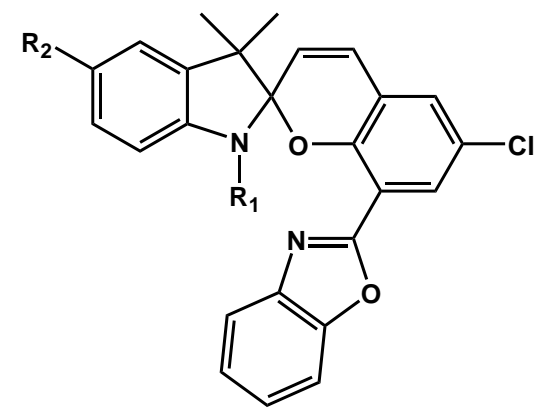

CП 4: $\mathrm{R}_{1}=\mathrm{CH}_{3}, \mathrm{R}_{2}=\mathrm{Cl}$

CП 5: $\mathrm{R}_{1}=\mathrm{CH}_{3}, \mathrm{R}_{2}=\mathrm{Br}$

CП 6: $\mathrm{R}_{1}=\mathrm{Pr}, \mathrm{R}_{2}=\mathrm{Br}$

CП 7: $\mathrm{R}_{1}=\mathrm{CH}_{2} \mathrm{Ph}, \mathrm{R}_{2}=\mathrm{H}$
Подобные отрицательные фотохромные превращения испытывают металлокомплексы 8-бензтиазолсодержащих производных СП 8-СП 13 [9] и 8-(5-(р-толил)-1,3,4-оксадиазол-2-ил) замещенных спиропиранов СП 14-СП - 18 [10], а также.<smiles>CCCCN1c2ccc(Br)cc2C(C)(C)C12C=Cc1cc(Cl)cc(-c3nc4ccccc4s3)c1O2</smiles>

$\mathrm{C \Pi}$ 8: $\mathrm{R}_{1}=\mathrm{CH}_{2} \mathrm{Ph}, \mathrm{R}_{2}=\mathrm{H}$

CП 9: $\mathrm{R}_{1}=\mathrm{CH}_{3}, \mathrm{R}_{2}=\mathrm{Br}$

CП 10: $\mathrm{R}_{1}=\mathrm{CH}_{3}, \mathrm{R}_{2}=\mathrm{Cl}$

CП 11: $\mathrm{R}_{1}=\mathrm{CH}_{3}, \mathrm{R}_{2}=\mathrm{CF}_{3}$

CП 12: $\mathrm{R}_{1}=\mathrm{CH}_{3}, \mathrm{R}_{2}=\mathrm{NO}_{2}$

CП 13: $\mathrm{R}_{1}=\mathrm{Pr}, \mathrm{R}_{2}=\mathrm{Br}$<smiles>[R8]c1cc2c(c(-c3nnc(-c4ccc(C)cc4)o3)c1)OC1(C=C2)N([R])c2ccc([R6])cc2C1(C)C</smiles>

CП 14: $\mathrm{R}_{1}=\mathrm{CH}_{3}, \mathrm{R}_{2}=\mathrm{R}_{3}=\mathrm{H}$

CП 15: $\mathrm{R}_{1}=\mathrm{CH}_{3}, \mathrm{R}_{2}=\mathrm{H}, \mathrm{R}_{3}=\mathrm{CHO}$

CП 16: $\mathrm{R}_{1}=\mathrm{CH}_{3}, \mathrm{R}_{2}=\mathrm{Cl}, \mathrm{R}_{3}=\mathrm{CHO}$

CП 17: $\mathrm{R}_{1}=\mathrm{CH}_{3}, \mathrm{R}_{2}=\mathrm{CF}_{3}, \mathrm{R}_{3}=\mathrm{CHO}$

CП 18: $\mathrm{R}_{1}=\mathrm{CH}_{2} \mathrm{Ph}, \mathrm{R}_{2}=\mathrm{H}, \mathrm{R}_{3}=\mathrm{CHO}$

Для спиропиранов СП 19 и СП 20 отрицательный фотохромизм обнаружен для протонированных комплексов этих соединений в водно-ацетонитрильных растворах [11].

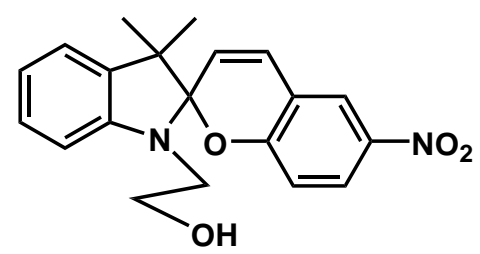

СП 19

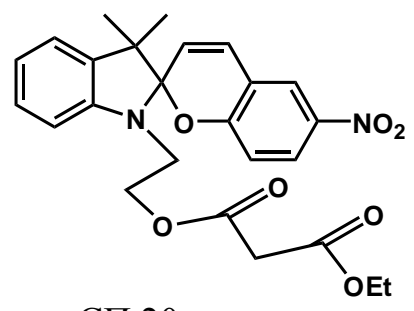

СП 20

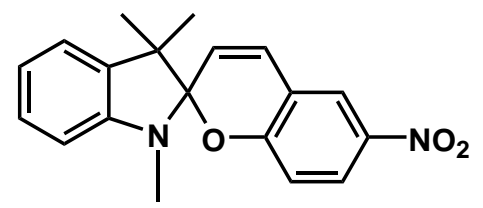

СП 21
Отрицательный фотохромизм протонных комплексов наблюдался при исследовании взаимодействия молекл спиропираноа СП 21 с поверхностью мезопористых наночастиц диоксида кремния
[12]. Такие наночастицы получали фотоиндуцированной адсорбцией спиропиранов в толуольном растворе $[12,13]$. Молекулы спиропирана на поверхности таких наночастиц присутствуют в мероцианиновой форме и проявляют отрицательный 
фотохромизм [14]. Подобный результат получен для дендридного волокнистого диоксида кремния [15]. Показано, что скорость темновой релаксации фотоиндуцированной циклической формы спиропирана в окрашенную мероциааниновую форму возрастает с увеличением размера пор наночастиц диоксида кремния [16].

С использованием спиропирана СП 21 были получены органофильные глины, проявляющие отрицательный фотохромизм [17].

Ряд исследований был направлены на синтез спиропиранов, структура которых обеспечивает проявление отрицательного фотохромизма. Спиропиран бнзофеназинового типа СП 22 проявляет в растворах как положительный, так и отрицательный фотохромизм [18].<smiles>CN1c2ccccc2C(C)(C)C12C=CC1=C(c3ccccc3Cl)OC12C</smiles>

В процессе синтеза индолиновых спиро-пиранов, содержащих сопряженные катионные винил-3Н-индолиновые фрагменты и их аналогов с формильным заместителем в 2 Н-хроменовом фрагменте получено соединение СП 23, проявляющее отрицательный фотохромизм под действием УФ излучения [19]. Пиридиний-содержащий спиропиран СП 24 проявляет отрицательный фотохромизм в растворах и положительный фотохромизм в кристаллическом состоянии [20].

Отрицательный фотохромизм обнаружен для сульфо-замещенных спиропиранов СП 25 - СП 27 [21]. Полоса поглощения исходной мероцианиновой формы этих соединений в диметилсульфоусиде расположена в коротковолновой спектральной области 350-450 нм. Спонтанное восстановление мероцианиновой формы из фотоиндуцированной формы составляет около 20 мин.

Спектрально-кинетическим методом изучен отрицательный фотохромизм пиридин-содержащих нитро-замещенных спиропиранов СП 28 СП 29 в растворах, твердофазных и полимерных слоях, а также в составе комплексов с катионами тербия [22]. Все исследованные соединения в водных растворах проявляют сходные фотохромные превращения. Наиболее эффективный отрицательный фотохромизм наблюдается для спиропирана СП 28. Увеличение длины заместителя R смещает равновесие мероцианиновой формы в сторону образования спирановой формы и снижает эффективность фотохромных превращений, возможно, вследствие стерических препятствий фотоиндуцированным процессам цис-транс-изомеризации. Эти соединения проявляют отрицательный фотохромизм как в твердофазных, так и в полимерных слоях. В присутствии катионов тербия длинноволновая полоса поглощения мероцианиновой формы спиропирана СП 28 испытывает гипсохромное смещение на 75 нм. При этом отрицательные фотохромные превращения сохраняются. Пиридин-замещенный спиропиран СП 30 проявляет отрицательный фотохромизм не только в полярных растворителях, но и в Ленгмюровских пленках [23].<smiles>[R]c1ccc(O)c(/C=C/C2=[N+](C)c3cc(S(=O)(=O)[O-])ccc3C2(C)C)c1</smiles>

$\mathrm{C} \Pi$ 25: $\mathrm{R}=\mathrm{H}$

$\mathrm{C} \Pi$ 26: $\mathrm{R}=\mathrm{CH}_{3}$

$\mathrm{C} \Pi$ 27: $\mathrm{R}=\mathrm{NO}_{2}$<smiles>[R]N1c2ccccc2C(C)(C)C12C=Cc1cc([N+](=O)[O-])cc(C[n+]3ccccc3)c1O2</smiles>

CП 28: $\mathrm{R}=\mathrm{C}_{8} \mathrm{H}_{17}$

CП 29: $\mathrm{R}=\mathrm{C}_{12} \mathrm{H}_{25}$

$\mathrm{C}$ П 30: $\mathrm{R}=\mathrm{C}_{18} \mathrm{H}_{37}$

Для получения систем с отрицательным фотохромизмом использовались фотохромные полимеры. К их числу относятся водорастворимые фотохромные полимеры с фрагментами незамещенного индолинового спиропирана [24]. Механизм негативных фотохромных превращений этих фотохромных полимеров основан на фотоиндуцированной депротонизации протонных комплексов. 
Спектральные и скоростные свойства содинений зависят от структуры спиропирановых фрагментов и заряда сомономеров. Отрицательный фотохромизм фотохромного сополимера, содержащего фрагменты спиропирана СП 21, обусловлен тем, что стабилизация мероцианиновой формы достигается меж- и внутримолекулярными взаимодействиями между индолениновым катионом и $\mathrm{COO}^{-}$анионом [25].

Для реализации ряда практических задач были синтезированы фотохромные полимеры с новыми функциональными свойствами. К их числу относятся сополимеры нитрозамещенного спиропирана с солевым $\mathrm{Ti}^{+4}$ - содержащим мономером, проявляющие отрицательный фотохромизм [26]. Эти фотохромные полимеры были использованы для получения металломицелл, обеспечивающих фотоуправляемое сульфоокисление веществ в воде с использованием перикиси водорода [26].

Для использования в механохромных устройствах были синтезированы фотохромные полимеры, в которых молекулы 3 спиропиранов СП 31 - СП 33 были ковалентно связаны с полярным поли(гидроксиэтил акрилатом) [27].<smiles>C=C(C)C(=O)Oc1ccc2c(c1)C(C)(C)C1(C=Cc3cc([N+](=O)[O-])cc(OC(=O)C(=C)C)c3O1)N2C</smiles>

СП 31

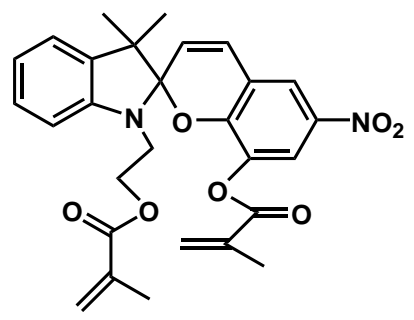

СП 32<smiles>C=C(C)C(=O)OCCN1c2ccc(OC(=O)C(=C)C)cc2C(C)(C)C12C=Cc1cc([N+](=O)[O-])cc(OC(=O)C(=C)C)c1O2</smiles>

СП 33
Стенхаус соединения

Фотохромные соединения этого типа, проявляющие отрицательный фотохромизм, интенсивно изучаются с 2014 г. в связи с перспективами их применения в биомедицинских исследованиях [28]. Они содержат в своей структуре электронодорный и электроноакцепторный фрагменты, которые обеспечивают фотоиндуцированную трансцис изомеризацию (схема 2).

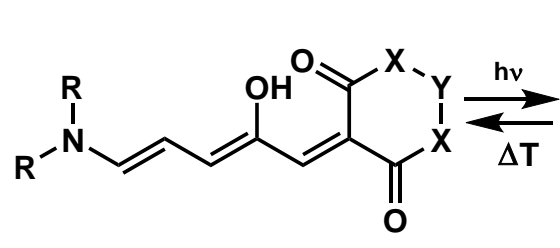

A<smiles>[X]C(=O)C(C([X])=O)[C@H]1C(=O)C=C[C@@H]1N([R])[R]</smiles>

B

Схема 2

Исследование отрицательного фотохромизма этих соединений показало зависимость спектральных характеристик от структуры соединений [29-31]. Полосы поглощения исходной транс-формы Стенхаус соединений могут быть батохромно сдвинуты путем изменения природы акцепторного фрагмента (рис. 2) [32].

Помимо спектральных свойств обнаружена зависимость скорости темнового окрашивания от природы акцептора [32]. С увеличением полярности растворителя концентрация транс-формы
Стенхаус соединений снижается, а концентрация цис-формы возрастает [28].<smiles>[X]C1=CC(=CC(O)=CC=CN2c3ccccc3CC2C)C(=O)P1</smiles>

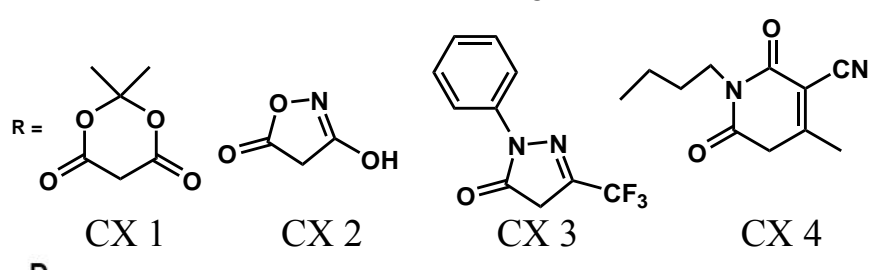

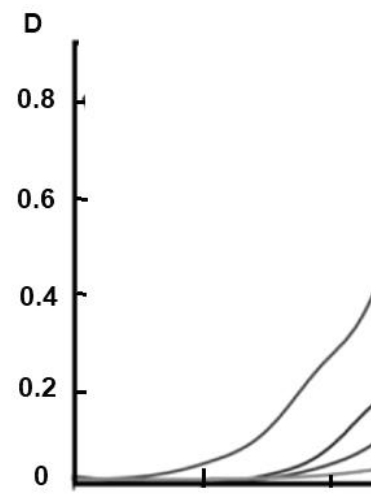

500
Рис. 2. Спектры поглощения исходной формы Стенхауз соединений СX 1 (1), СX 2 (2), СX 3 (3), СX 4 (4) в воде

Подобными фотохромными превращениями обладают полимерные Стенхаус соединения CX 5 - CX 8 [33]. 
<smiles>CNCCCN(/C=C/C=C(\O)C=C1C(=O)OC(C)(C)OC1=O)c1ccc(OC)cc1</smiles>

CX 5<smiles>CNCCCN(/C=C/C=C(\O)C=C1C(=O)N(C)C(C)(C)N(C)C1=O)c1ccc(OC)cc1</smiles>

CX 7

Для соединения CX 9 показано, что наряду с фотоиндуцированным изменением полос поглощения наблюдается фотоиндуцированная модуляция флуоресценции [34].<smiles>[R4]CCN1CCc2cc(-c3ccc4c5c(cccc35)C(=O)N(CCCC)C4=O)ccc2N1/C=C/C=C(\O)C=C1C(=O)N(C)C(=O)N(C)C1=O</smiles>

Важное значение имеет наличие гидроксильной групп в полиеновой цепи, которые стабилизируюет транс-форму этого соединения за счет образования водородной связи [34]. Это подтверждается спектрально-кинетическим методом сравнительного исследования с аналогами соединений, не содержащих гидроксильных групп и квантовохимическими расчетами. Экспериментальное и теоретическое исследование сольватохромизма Стенхауз соединений показало, что для улучшения их функционаьных свойств необходимо детально исследовать темновые процессы релаксации и снизить влияние сольватации на их скоростные харатеристики [35].

Изменение спектров поглощения и флуоресценции наблюдается при введении в водные растворы кислот и солей металлов $[36,37]$. Полимерные слои на их основе могут использоваться для определения содержания аминов и в качестве индикаторов температуры [38].<smiles>CC1(C)OC(=O)C(=C/C(O)=C/C=C/N2CC(CCN[Al+])c3ccccc32)C(=O)O1</smiles>

CX 6<smiles>CN1C(=O)C(=C/C(O)=C/C=C/N2CC(CCN[As])c3ccccc32)C(=O)N(C)C1(C)C</smiles>

CX 8

Для создания роботизированных систем предлагается использовать полимерные бислои, состоящие из полиимидной пленки, на которую нанесено полимерное Стенхаус соединение [39]. Такие пленки способны изменять свою конформацию при облучении видимым светом.<smiles>CC1Cc2ccccc2N1/C=C/C=C(O)/C=C1\C(=O)N(c2ccccc2)N=C1C(F)(F)F</smiles>

Стенхаус соединение СX 10 использовалось для изучения потоков в жидких растворах [40].

\section{Нитрил-содержащие соединения}

Отрицательный фотохромизм проявляют соединения, полученные в результате взаимодействия трицианофуранов или гидрокситрицианопиррола с метокси-замещенными 2-гидроксибензальдигидами (схема 3) [41].<smiles>CC1(C)OC(=C(C#N)C#N)C(C#N)=C1/C=C/c1cccc2ncccc12</smiles> 
<smiles>N#CC(C#N)=C1OC(c2ccccc2)(c2ccccc2)C(C(F)(F)F)=C1C#N</smiles>

HC 2<smiles>CC1(C)OC(=C(C#N)C#N)C(C#N)=C1C=Cc1ccccc1O</smiles>

HC 3
Одно из первых соединений этого типа НС 1 характеризовалось полосой поглощения с максимумом при 428 нм [41]. Подобные фотохромные превращения испытывает соединение НС 2 (рис. 3), но максимум полосы его поглощения батохромно смещается до 474 нм [42]. Отрицательный фотохромизм проявляют соединения НС 3 как метаноле, так и в полимерных слоях [43].

В результате спектрально-кинетического<smiles>[X]C([R])(C)C(/C=C/c1ccccc1OC)=C(C#N)C(C#N)=C(C#N)C#N</smiles>

$\mathrm{HC} 4$<smiles>[R]c1cc([R1])c(O)c(/C=C/C2=C(C#N)C(=C(C#N)C#N)OC2(C)C)c1</smiles>

$\mathrm{HC}$ 6: $\mathrm{R}^{1}=\mathrm{H} ; \mathrm{R}^{2}=\mathrm{NO}_{2}$

$\mathrm{HC}$ 7: $\mathrm{R}^{1}=\mathrm{NO}_{2} ; \mathrm{R}^{2}=\mathrm{H}$

$\mathrm{HC}$ 8: $\mathrm{R}^{1}=\mathrm{NO}_{2} ; \mathrm{R}^{2}=\mathrm{NO}_{2}$

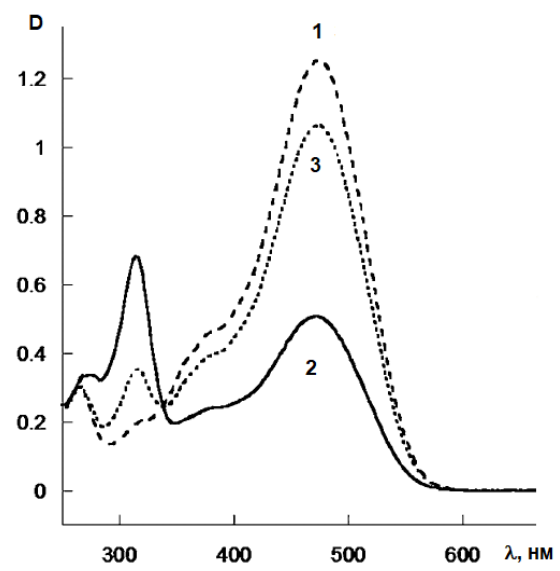

Рис. 3. Изменение спектров поглощения соединения НС 2 в этаноле до (1), после облучения видимым светом в течение 5

c (2) и в процессе темновой релаксации через 5 с после оотключения света (3) исследования серий соединений НC 4 и НС 5 в зависимости от их структуры и природы заместителей установлено, что положение максимумов полос поглощения исходной формы А изменялись в диапазоне 390-565 нм, а значения констант скорости темновой релаксации обесцвеченной формы В - от $\mathrm{k}=10^{-1}$ до $10^{-9} \mathrm{c}^{-1}$ [44-46]. Соединения этого типа характеризуются и высокой цикличностью фотохромных превращений.<smiles>[R]c1ccc(O)c(/C=C/C2=C(C#N)C(=C(C#N)C#N)C([Z])(C)C2=[X])c1</smiles>

HC 5

Соединения НС 6 - НС 8 обладают обратимыми не только абсорбционными, но и люминесцентными свойствами [47].

Биимидазолильные радикальные комплексы Впервые отрицательный фотохромизм (схема 4) была обнаружен у соединений БИ 1 БИ 3 [48].

Отличительной особенностью этих соединений является миллисекундное время существования фотоизомеров при обычных температурах.

Соединения БИ 4 и БИ 5 характеризуются полосой поглощения исходной формы в видимой области спектра 380-480 нм [49]. В отличие от предыдущих соединений время релаксации этих соединений в обезгаженном бензоле составляет для соединения БИ 410,9 мин при $40^{\circ} \mathrm{C}$, а для БИ 5 - 1,9 с при $25^{\circ} \mathrm{C}$.

Область спектральной чувствительности биимидазолильных комлексов удалось сдвинуть в длинноволновую спектральную область путем введения электроно-донорных заместителей в структуру бинафтил-мостиковых биимидазольных димеров БИ 6 и БИ 7 (рис. 4) [50]. В зависимости от структуры соединений и природы заместителей положение максимумов полос поглощения меняется в спектральном диапазоне 490-646 нм, а время темновой релаксации фотообесцвеченной формы 
до достижения половины исходной оптической плотности - от 30 с 2,7 мин. Для совершенствования свойств соединений этого типа, обладающих отрицательным фотохромизмом, разработан квантово-химический метод расчета структур с ожидаемыми свойствами [51].<smiles>O=C1C=CC2(C=C1)c1cccc3cccc(c13)-c1nc(-c3ccccc3)c(-c3ccccc3)n12</smiles>

A<smiles>O=C1C=CC2(C=C1)c1cccc3cccc(c13)-c1nc(-c3ccccc3)c(-c3ccccc3)n12</smiles>

БИ 1

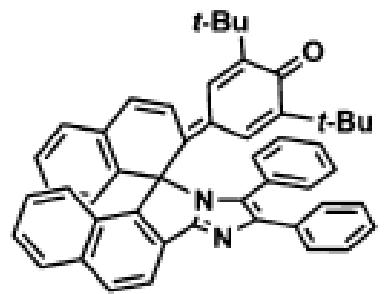

БИ 4

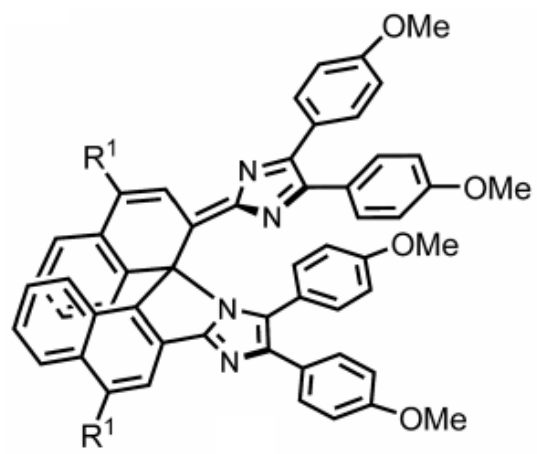

БИ 6

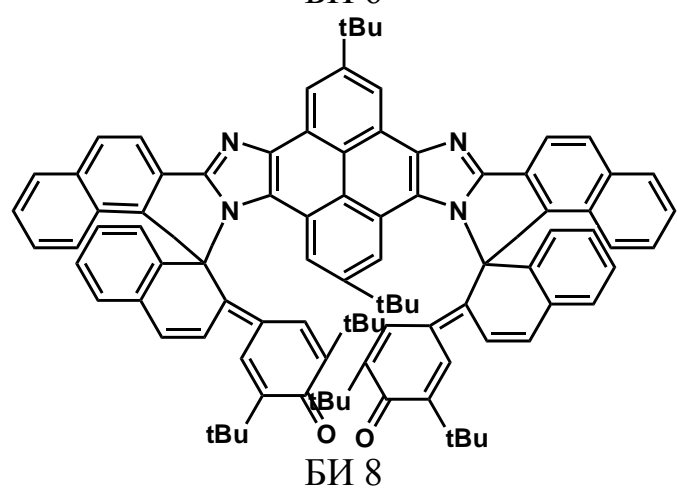

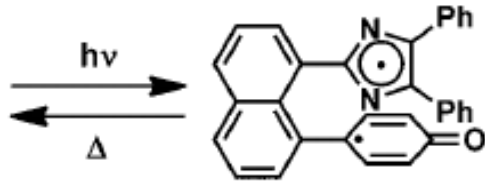

B

Схема 4

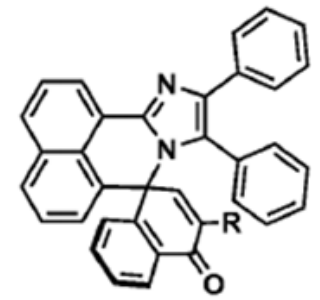

БИ 2: $\mathrm{R}=\mathrm{H}$;

БИ 3: $\mathrm{R}=\mathrm{t}-\mathrm{Bu}$

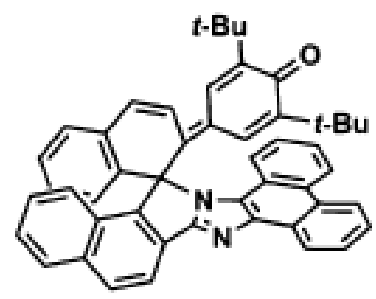

БИ 5

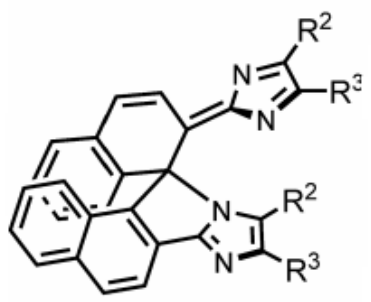

БИ 7

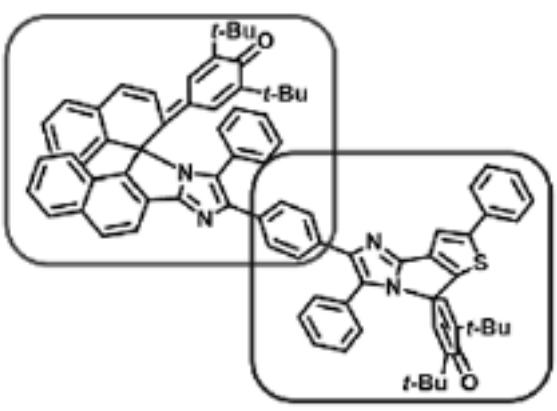

БИ 9 


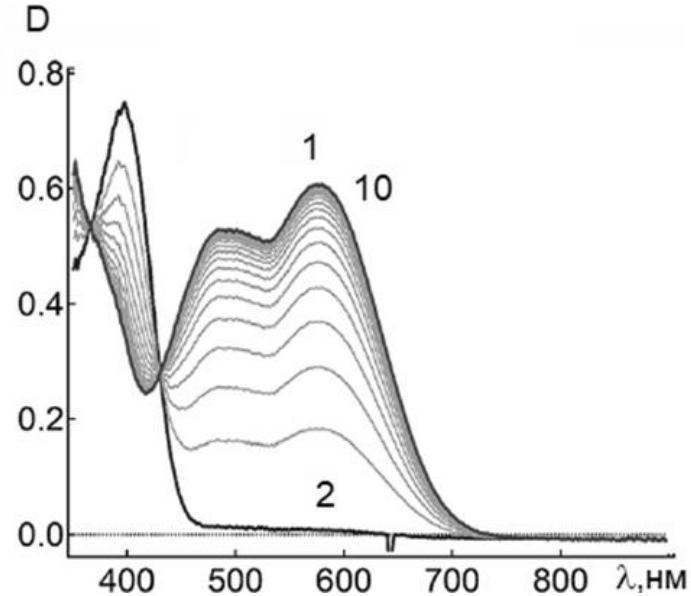

Рис. 4. Изменение спектров поглощения соединения из серии БИ 6 в обезгаженном бензоле при 298 К до (1), после облучения видимым светом (642 нм, 34 мВт) (2) и в процессе темовой релаксации (3-10)

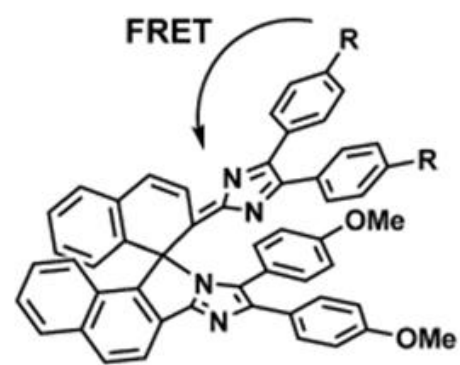

БИ 10
Для бихромофорного соединения БИ 8 обнаружена нелинейная зависимость фотопревращения от интенсивности обесцвечивающего видимого излучения [52]. Бихромофорное соединение БИ 9 проявляет как отрицательный, так и положительный фотохромизм [53].

Введение в структуру биимидазолильного димера органического флуорофора (нафталимида) позволило создать фотохромные соединения БИ 10 с фотоиндуцированной модуляцией флуоресценями [54]. Фотоиндуцированная модуляция флуоресценции достигается за счет индуктивно-резонансного переноса энергии от флуорофорного фрагмента на соединение в исходном окрашенном состоянии (FRET-эффект). Флуоресценция возникает после фотообесцвечивания раствора.

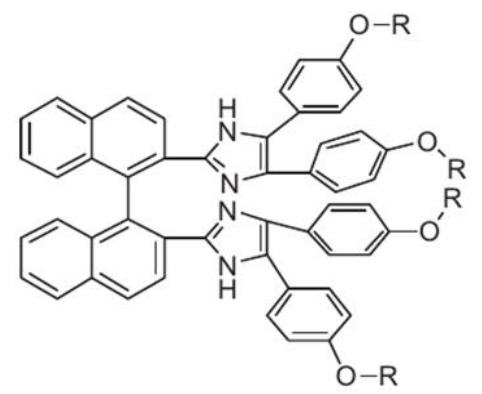

БИ 11 ции в соответствии с фотохромными превращени-

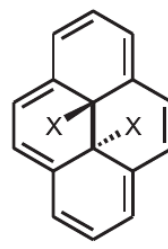<smiles>C1=C[C@H]2C[C@@H]1C2</smiles>

Схема 5

Соединения этого типа БИ 11 были использованы для исследования возможности создания фотоуправляемых средств доставки лекарств с жидкокристаллическими фрагментами [55].

Дигидропирены

Соединения этого типа проявляют отрицательный фотохромизм в результате обратимой валентной изомеризации (схема 5) [1].

Отрицательный фотохромизм проявляет соединение ДГП 1, поглощающее в области 450550 нм. Оно обесцвечиваясь под действием видимого излучения и возвращаясь в исходное состояние при нагревании при $50{ }^{\circ} \mathrm{C}$. Показано, что это соединение может использоваться для фотоуправления уровнями электронной энергии в оптоэлектронных устройствах [56].

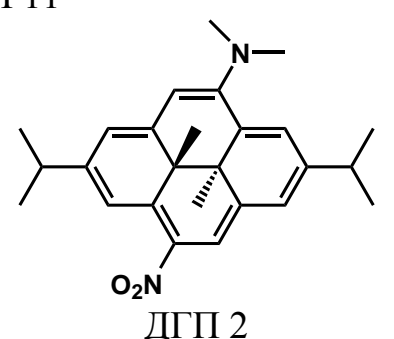

ДГП 1

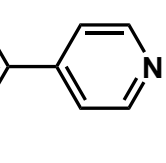

Наиболее существенным результатом синтетических исследований является получение соединения ДГП 2, проявляющего отрицательный фотохромизм, с длинноволновой полосой поглощения, которая располагается в биологически важном длинноволновом спектральном диапазоне (рис. 5) [57].

Для целенаправленного синтеза дигидропиренов с отрицательным фотохромизмом разработаны квантово-хмические методы определения их спектрально-кинетических характеристик [58]. Проведены квантово-химические расчеты нелинейно-оптических сойств фотохромных дигидропиренов с целью создания эффективных фотоуправляемых оптических материалов [59]. 
V.A. Barachevsky

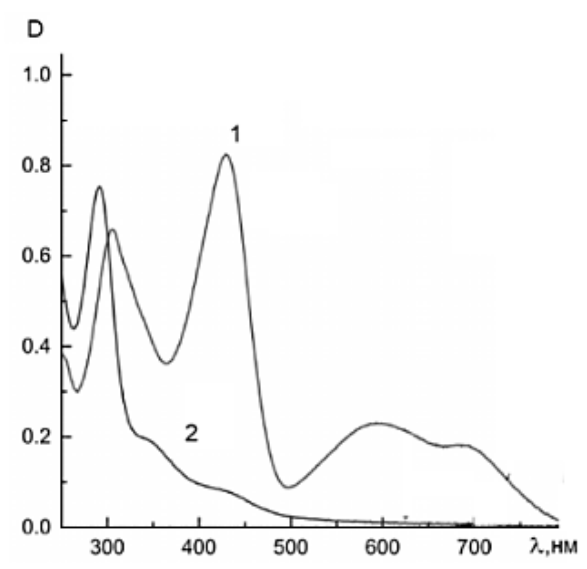

Рис. 5. Спектры поглощения соединения ДГП 2 в $\mathrm{CH}_{3} \mathrm{CN}$ до (1) и после воздействия лазерного диода с $\lambda=780$ нм (2)

Другие соединения

Отрицательный фотохромизм проявляет цианиновый краситеь Д 1 (схема 6, рис. 6) [60].

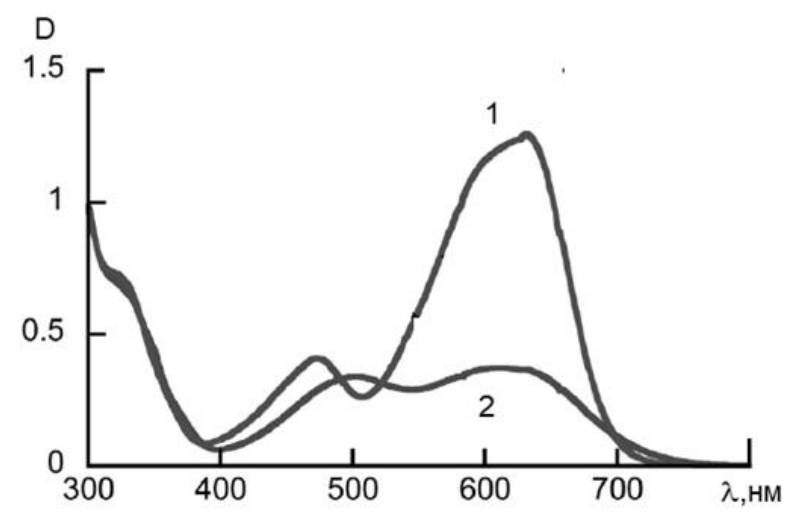

Рис. 6. Спектры поглощения соединения Д 1 в ацетонитриле до (1) и после облучения видимым свтом (2)

Протонированная форма азулена Д 2, напоминающая открытую форму диарилэтена, проявляет отрицательный фотохромизм в циклогексане (схема 7, рис. 7) [61].

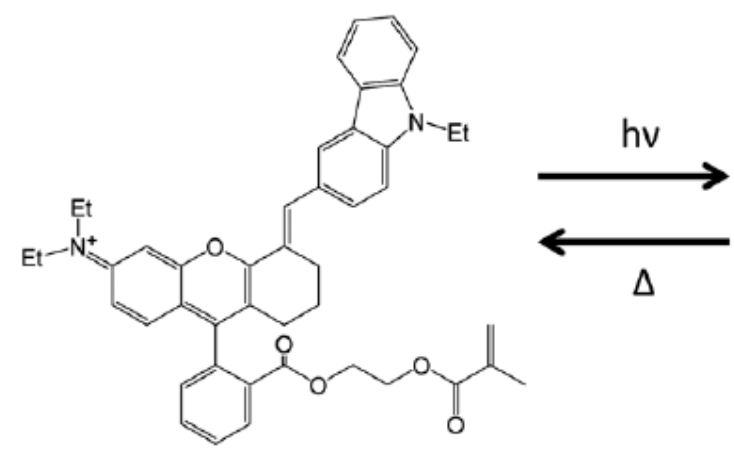

A

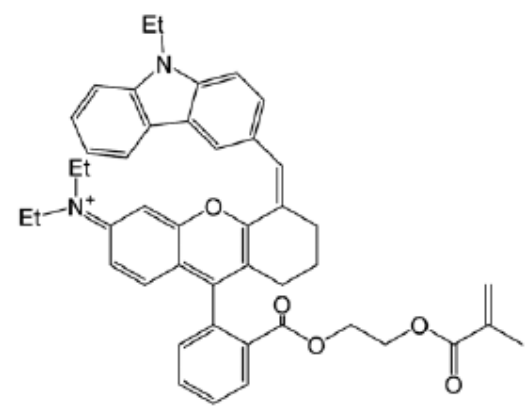

B

Схема 6

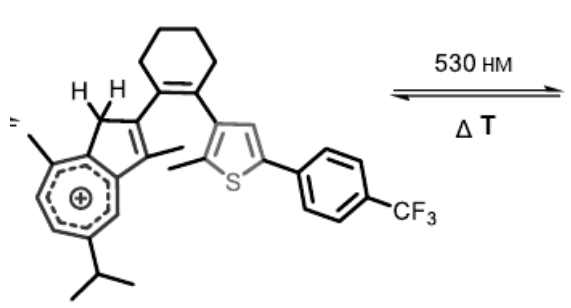

A

Д 2

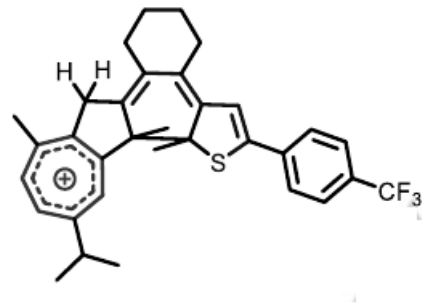

B

Схема 7<smiles></smiles>

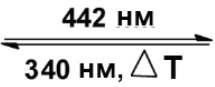<smiles>CCOC(=O)/C(=N/N[C]1CN2C=C1C=CC2)c1ccccc1</smiles>

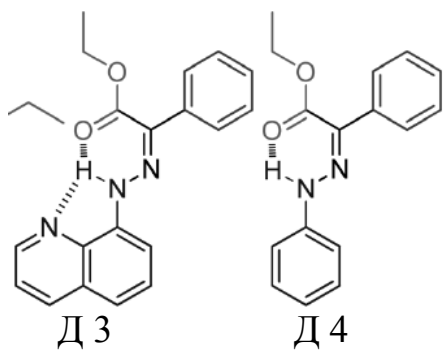

Схема 8

15 


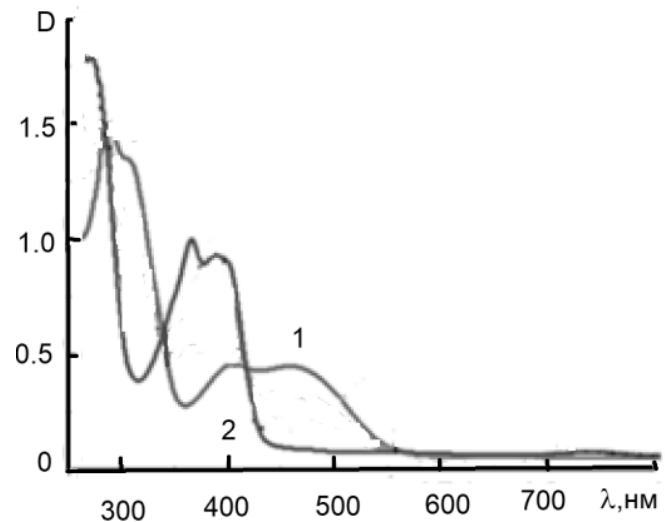

Рис. 7. Изменение спектров поглощения соединения Д 2 в циклогексане до (1) и после (2) облучения видимым светом при температуре $-30{ }^{\circ} \mathrm{C}$

В случае гидразонов Д 3 и Д 4 отрицательный фотохромизм (схема 8 , рис. 8) проявляется в гипсохромном сдвиге полосы поглощения [62].

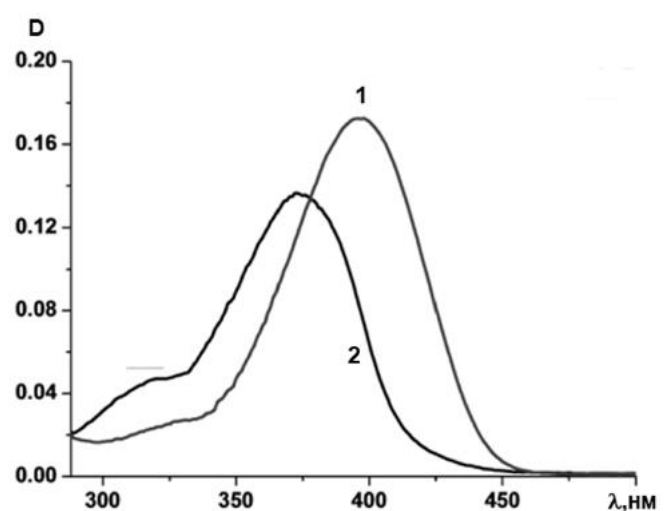

Рис. 8. Спектры поглощения соединения Д 3 в толуоле до (1) и после облучения видимым свтом с $\lambda=442$ нм (2)

\section{ВЫВОДЫ}

Исследование явления отрицательного фотохромизма становится одним из важных направлений развития фотохромизма органических соединений. Наибольшее внимание уделяется синтезу и исследованию свойств фотохромных систем на основе функционализированных спиропиранов и их комплексов с ионами металлов и протонами.

В отличие от фотохромных спиропиранов синтез новых Стенхаус соединений и нитрил-содержащих фотохромов обеспечивает получение фотохромных систем с различными спектрами поглощения (окрасками), зависящими от структуры соединений и природы заместителей.

Получены биимидазолильные радикальные комплексы с отрицательным фотохромизмом, отличающиеся от рассмотренных выше фотохромных соединений, высокой скоростью релаксации фотоиндуцированной формы в исходное состояние.
Перспективными представляются пока еще немногочисленные синтетические исследования цианиновых красителей, азуленов и гидразонов, проявляющих отрицательный фотохромизм.

Залогом дальнейшего развития исследований в этой области фотохромизма является практическая востребованность фотохромных материалов с обратным фотохромизмом для создания покрытий с фотодинамическим изменением окраски и инновационных средств для биомедицинских технологий.

Работа выполнена при поддержке Министерства науки и высшего образования в рамках выполнения работ по Государственным заданиям ФНИЦ «Кристаллография и фотоника» РАН и МЦАИ РАН.

\section{ЛИТЕР А Т У Р А}

\section{REFERENCES}

1. Photochromic Materials: Preparation, Properties and Applications. Tian H. and Zhang J. (Eds.), Weinheim, Germany:Wiley-VCH Verlag GmbH \& Co. KGaA. 2016.421 p.

2. Applied Photochromic Polymer Systems. McArdle C.B. (Ed.). Glasgow: Blackiev \&Son Ltd. 1991. 255 p.

3. Barachevsky V.A., Krayushkin M.M., Kiyko V.V. Light-Sensitive Organic Recording Media for Three-Dimensional Optical Memory. In: Photon-Working Switches. Eds. Yokoyama Y., Nakatani K. Springer Japan KK. 2017. P. 181-207.

4. Barachevsky V.A. Negative Photochromism in Organic Systems. Rev. J. Chem. 2017. V. 7. P. 334-371. DOI: 10.1134/S2079978017030013.

5. Aiken S., Edgar R.J.L., Gabbutt C.D., Heron B.M., Hobson P.A. Negatively photochromic organic compounds: Exploring the dark side. Dyes and Pigments. 2018. V. 149. P. 92-121. DOI: 10.1016/ j.dyepig.2017. 09.057.

6. Барачевский В.А., Валова Т.М. Спектрально-кинетическое исследование отрицательного фотохромизма систем на основе комплексов спиропиранов с ионами металлов. Оптика и спектроскопия. 2017. Т. 123. № 3. C. 377-383. DOI: 10.1134/S0030400X17090065. Barachevsky V.A., Valova T.M. A spectral-kinetic investigation of the negative photochromism of systems based on complexes of spiropyrans with metal ions. Opt. Spectr. 2017. V. 123. N 3. P. 404-410. DOI: 10.1134/S0030400X17090065.

7. Барачевский В.А., Валова Т.М. Флуоресцентные свойства полимерных систем с отрицательным фотохромизмом на основе комплексов спиропирана с ионами металлов. Ж. прикл. химии. 2021. Т. 94. № 3. С. 44-49. Barachevsky V.A, Valova N.M. Fluorescence properties of polymeric systems with negative phochromism based on complexes of spiropyran with metal ions. Rus. J. Appl. Chem. 2021. T. 94. N 3. P. 44- 49. DOI: $10.1134 / \mathrm{S} 1070427221030034$.

8. Chernyshev A.V., Metelitsa A.V., Rostovtseva I.A., Voloshin N.A., Solov'eva E.V., Gaeva E.B., Minkin V.I. Chromogenic systems based on 8-(1,3-benzoxazol-2-yl) substituted spirobenzopyrans undergoing ion modulated photochromic rearrangements. J. Photochem. Photobiol. A. 2018. V. 360. P. 174-180. DOI: 10.1016/ j.jphotochem. 2018.04.031.

9. Chernyshev A.V., Voloshin N.A., Rostovtseva I.A., Demidov O.P., Shepelenko K.E., Solov'eva E.V., Gaeva E.B., Metelitsa A.V. Benzothiazolyl substituted spiropyrans with ion-driven 
photochromic transformation. Dyes and Pigments. 2020. V. 178. Art. 108337. DOI: 10.1016/j.dyepig.2020.108337.

10. Chernyshev A.V., Voloshin N.A., Solov'eva E.V., Gaeva E.B., Zubavichus Y.V., Lazarenko V.A., Vlasenko V.G., Khrustalev V.N., Metelitsa, A.V. Ion-depended photochromism of oxadiazole containing spiropyrans. J. Photochem. Photobiol. A. 2019. V. 378 P. 201-210. DOI: 10.1016/j.jphotochem.2019.04.037.

11. Валова Т.М., Барачевский В.А., Хузин А.А., Туктаров $A . P$. Отрицательный фотохромизм водно-ацетонитрильных растворов функционализированных спиропиранов. Журн. общ. хим. 2019. Т. 89. № 9. С. 1375-1378. DOI: 10.1134/S0044460X19090099. Valova T.M., Barachevsky V.A., Khuzin A.A., Tuktarov A.R. Negative photochromism of solutions of functionalized spiropyrans in a water - acetonitrile mixture. Rus. J. General Chem. 2019. V. 89. P. 1783-1786. DOI: 10.1134/ S0044460X19090099.

12. Okabe Y., Ogawa M. Photoinduced adsorption of spiropyran into mesoporous silicas as photomerocyanine. RSC Adv. 2015. V. 5. P. 101789-101793. DOI: 10.1039/ C5RA18252B.

13. Yamaguchi T., Maity A., Polshettiwar V., Ogawa M. Photochromism of a spiropyran in the presence of a dendritic fibrous nanosilica; simultaneous photochemical reaction and adsorption. J. Phys. Chem. A. 2017. V. 121. P. 8080-8085. DOI: $10.1021 /$ acs.jpca.7b08466.

14. Yamaguchi, T., Ogawa, M. Hydrophilic internal pore and hydrophobic particle surface of organically modified mesoporous silica particle to host photochromic molecules. Chem. Lett. 2019. V. 48. N 2. P. 170-172. DOI: 10.1246/cl.180908.

15. Yamaguchi T., Maity A., Polshettiwar V., Ogawa M. Negative photochromism based on molecular diffusion between hydrophilic and hydrophobic particles in the solid state. Inorg. Chem. 2018. V. 57. N 7. P. 3671-3674. DOI: 10.1021/acs.inorgchem.7b03132.

16. Yamaguchi T., (Nut) Leelaphattharaphan N., Shin H., Ogawa M. Acceleration of photochromism and negative photochromism by the interactions with mesoporous silicas. Photochem. Photobiolog. Sci. 2019. V. 18. P. 1742-1749. DOI: 10.1039/c9pp00081j.

17. Yamaguchi T., Imwiset K. J., Ogawa M. Efficient negative photochromism by the photoinduced migration of of photochromic merocyanine/spiropyran in the solid state. Langmuir. 2021. V. 37. N 12. P. 3702-3708. DOI: 10.1021/acs.langmuir.1c00150.

18. Metelitsa A., Chernyshev A., Voloshin N., Solov'eva E., Rostovtseva I., Dorogan I., Gaeva E., Guseva A. Semipermanent merocyanines of spirocyclic compounds: photochromic "balance. Dyes and Pigments. 2021. V. 186. Art. 109070. DOI: 10.1016/j.dyepig.2020.109070.

19. Pugachev A.D., Ozhogin I.V., Lukyanova M.B., Lukyanov B.S., Kozlenko A.S., Rostovtseva I.A., Makarova N.I., Tkachev V.V., Aldoshin S.M., Metelitsa A.V. Synthesis, structure and photochromic properties of indoline spiropyrans with electron-withdrawing substituents. J. Molec. Struct. 2021. V. 1229. Art. 129615. DOI: 10.1016/j.molstruc.2020.

20. Funasako Y., Miyazaki H., Sasaki T., Goshima K., Inokuchi M. Synthesis, photochromic properties, and crystal structures of salts containing a pyridinium-fused spiropyran: positive and negative photochromism in the solution and solid state. J. Phys. Chem. B. 2020. V. 124. P. 7251-7257. DOI: 10.1021/acs.jpcb.0c04994.

21. Gao H., Guo T., Chen Y., Kong Y., Peng Z. Reversible negative photochromic sulfo-substituted spiropyrans. J. Molecul. Struct. 2016. V. 1123. P. 426-432. DOI: 10.1016/ j.molstruc. 2016.07.050.

22. Барачевский В.А., Валова Т.М., Атабекян Л.С., Любимов $A . B$. Отрицательный фотохромизм водорастворимых пиридин-содержащих нитрозамещенных спиропиранов. Химия высоких энергий. 2017. Т. 51. № 6. С. 436-441. DOI: 10.1134/S0018143917060029. Barachevsky V.A., Valova
T.M., Atabekyan L.S., Lyubimov A.V. Negative photochromism of water-soluble pyridine-containing nitro-substituted spiropyrans. High Energy Chem. 2017. V. 51. N 6. P. 415-419. DOI: $10.1134 / \mathrm{S} 0018143917060029$.

23. Koryako N.E., Ivakhnenkoa D.A., Ivakhnenkoa A.A., Lyubimov A.V., Zaichenko N.L., Lyubimova G.V., Arslanova V.V., Shokurova A.V., Raitman O.A. Negative photochromism and luminescent properties of amphiphilic spiropyran in solutions and at the interface. Protect. Metals Phys. Chem. Surf. 2019. V. 55. N 6. P. 1118-1123. DOI: 10.1134/S2070205119060194.

24. Feeney M.J., Thomas S.W. Tuning the regative photochromism of water-soluble spiropyran polymers. Macromolecules. 2018. V. 51. N 20. P. 8027-8037. DOI: 10.1021/acs.macromol.8b01915.

25. Sun B.-B., Yao B.-H., Fu Z.-S., He Y.-Q. Preparation and analysis of photochromic behavior of carboxymethyl chitin derivatives containing spiropyran moieties. Design. Monom. Polymers. 2020. V. 23. N. 1. P. 106-117. DOI:10.1080/15685551.-2020.-1796362.

26. Tang Z., Wang W., Pi Y., Wang J., Li C., Tan R., Yin D. Visible light-controlled reaction-separation for asymmetric sulfoxidation in water with photo-responsive metallomicelles. ACS Sustain. Chem. Engineer. 2019. V. 7. P. 17967-17978. DOI: 10.1021/acssuschemeng.9b04712.

27. Qiu W., Gurr P.A., Qiao G.G. Color switchable polar polymeric materials. ACS Appl. Mater. Interfac. 2019. V. 11. No. 32. P. 29268-29275. DOI: 10.1021/acsami.9b09023.

28. Lerch M.M., Szymański W., Feringa B.L. The (photo)chemistry of Stenhouse photoswitches: guiding principles and system design. Chem. Soc. Rev. 2018. V. 47. N 6. P. 1910-1937. DOI: $10.1039 / \mathrm{c} 7 \mathrm{cs} 00772 \mathrm{~h}$.

29. Lerch M.M., Wezenberg S.J., Szymański W., Feringa B. Unraveling the photoswitching mechanism in donor-acceptor Stenhouse adducts. J. Am. Chem. Soc. 2016. V. 138. P. 6344-6347. DOI: 10.1021/jacs.6b01722.

30. Helmy S., Oh S., Leibfarth F.A., Hawker C.J., Read de Alaniz J. Design and synthesis of donor-acceptor Stenhouse adducts: a visible light photoswitch derived from furfural. J. Org. Chem. 2014. V. 79. P. 11316-11329. DOI: 10.1021/jo502206g.

31. Mallo N., Brown P.T., Iranmanesh H., MacDonald T.S.C., Teusner M.J., Harper J.B., Ball G.E., Beves J.E. Photochromic switching behaviour of donor-acceptor Stenhouse adducts in organic solvents. Chem. Commun. 2016. V. 52. P. 13576-13579. DOI: 10.1039/C6CC08079K.

32. Hemmer J.R., Page Z.A., Clark K.D., Stricker F., Dolinski N.D., Hawker C.J., Read de Alaniz J. Controlling dark equilibria and enhancing donor-acceptor Stenhouse adduct photoswitching properties through carbon acid design. J. Amer. Chem. Soc. 2018. V. 140. P. 10425-10429. DOI: $10.1021 / \mathrm{jacs} .8 \mathrm{~b} 06067$.

33. Ulrich S., Hemmer J.R., Page Z.A., Dolinski N.D., Rifaie-Graham O., Bruns N., Hawker C.J., Boesel L.F., Read De Alaniz. $J$. Visible light-responsive DASA-polymer conjugates. ACS Macro Lett. 2017. V. 6. P. 738-742. DOI: 10.1021/ acsmacrolett.7b00350.

34. Lerch M.M., Medved' M., Lapini A., Laurent A. D., Iagatti A., Bussotti L., Szymański W., Buma W.J., Foggi P., Di Donato M., Feringa B.L. Tailoring photoisomerization pathways in donoracceptor Stenhouse adducts: the role of the hydroxy group. J. Phys. Chem. A. 2018. V. 122. N 4. P. 955-964. DOI: 10.1021/acs.jpca. 7b10255.

35. Lerch M.M., Di Donato M., Laurent A.D., Medved' M., Iagatti A., Bussotti L., Lapini A., Buma W.J., Foggi P., Szymański W., Feringa B.L. Solvent effects on the actinic step of donor-acceptor Stenhouse adduct photoswitching. Angew. 
Chem. Intern. Ed. 2018. V. 57. N 27. P. 8063-8068. DOI: 10.1002/anie. 201803058.

36. Yang S., Liu J., Cao Z., Li M., Luo Q., Qu D. Fluorescent photochromic donor-acceptor Stenhouse adduct controlled by visible light. Dyes and Pigments. 2018. V. 148. P. 341-347. DOI: 10.1016/j.dyepig.2017.09.040.

37. Chen T.-Y., Cai Y.-D., Jiang S.-Q., Cai W.T., Ming-Liang, Bao $X$. Light- and chemical-stimuli-induced isomerization of donor-acceptor Stenhouse adducts. Chem. Photo Chem. 2021. DOI: $10.1002 /$ cptc.202100004.

38. Chen Q., Diaz Y.J., Hawker M.C., Martinez M.R., Page Z.A., Xiao-An Zhang S., Hawker C. J. Read de Alaniz J. Stable activated furan and donor-acceptor Stenhouse adduct polymer conjugates as chemical and thermal sensors. Macromolecules. 2019. V. 52. P. 4370-4375. DOI: 10.1021/acs.macromol.9b00533.

39. Lee J., Sroda M.M., Kwon Y., El-Arid S., Seshadri S., Gockowski L. F., Hawkes E.W., Valentine M.T., Read de Alaniz J. Tunable photothermal actuation enabled by photoswitching of donor-acceptor Stenhouse adducts. ACS Appl. Mater. Interf. 2020. V. 12. N 48. P. 54075-54082. DOI: 10.1021/acsami. $0 \mathrm{c} 15116$.

40. Seshadri S., Gockowski L.F., Lee J., Sroda M., Helgeson M.E., Read de Alaniz J., Valentine M.T. Self-regulating photochemical Rayleigh-Bénard convection using a highly-absorbing organic photoswitch. Nat. Commun. 2020. V. 11. Art.2599. DOI: $10.1038 / \mathrm{s} 41467-020-16277-7$.

41. Peng P., Strohecker D., Liao Y. Negative photochromism of a TCF chromophore. Chem. Commun. 2011. V. 47. N 30. P. 8575-8577. DOI: $10.1039 / \mathrm{c} 1 \mathrm{cc} 12379 \mathrm{c}$

42. Johns V.K., Peng P., DeJesus J., Wang Z., Liao Y. Visiblelight-responsive reversible photoacid based on a metastable carbanion. Chem. Eur. J. 2013. V. 20. N 3. P. 689-692. DOI: $10.1002 /$ chem. 201304226 .

43. Yang C., Khalil T., Liao Y. Photocontrolled proton transfer in solution and polymers using a novel photoacid with strong $\mathrm{C}-$ H acidity. RSC Adv. 2016. V. 6. N 8. P. 85420-85426. DOI: 10.1039/c6ra12966h.

44. Belikov M.Yu., Ievlev M.Yu., Fedoseev S.V., Ershov O.V. Novel group of negative photochromes containing a nitrilerich acceptor: synthesis and photochromic properties. Res. Chem. Intermed. 2019. V. 45. P. 4625-4636. DOI: 10.1007/s11164-019-03853-w.

45. Belikov M.Y., Ievlev M.Y., Fedoseev S.V., Ershov O.V. Tuning the photochromic properties of chromophores containing a nitrilerich acceptor: a novel branch in the investigation of negative photochromes. New J. Chem. 2019. V. 43. P. 8414-8417. DOI: 10.1039/c9nj01648a.

46. Belikov M.Y., Fedoseev S.V., Ievlev M.Y., Ershov O.V., Lipin K.V., Tafeenko V.A. Direct synthesis of variously substituted negative photochromes of hydroxytricyanopyrrole (HTCP) series. Synth. Commun. 2020. V. 50. N 16. P. 2413-2421. DOI: 10.1080/ 00397911.2020.1772822.

47. Belikov M.Yu., Ievlev M. Yu.,. Fedoseev S.V, Ershov O.V. The first example of "turn-off" red fluorescence photoswitching for the representatives of nitrile-rich negative photochromes. New J. Chem. 2020. V. 44. P. 6121-6124. DOI: 10.1039/D0NJ00718H.

48. Mutoh K., Kobayashi Y., Hirao Y., Kubo T., Abe J. Stealth fast photoswitching of negative photochromic naphthalene-bridged phenoxyl-imidazolyl radical complexes. Chem. Commun. 2016. V. 52. N 41. P. 6797-6800. DOI: 10.1039/C6CC01534D.
49. Yamaguchi T., Kobayashi Y., Abe J. Fast negative photochromism of 1,1'-binaphthyl-bridged phenoxyl-imidazolyl radical complex. J. Am. Chem. Soc. 2016. V. 138. N 3. P. 906-913. DOI: 10.1021/jacs.5b10924.

50. Kometani A., Inagaki Y., Mutoh K., Abe J. Red or NIR light operating negative photochromism of binaphthyl-bridged imidazole dimer. J. Amer. Chem. Soc. 2020. V. 142. P. 7995-8005. DOI: 10.1021/jacs.0c02455.

51. Abe J., Ito H., Mutoh K. Enhancement of negative photochromic properties of naphthalene- bridged phenoxyl- imidazolyl radical complex. ChemPhysChem. 2020. V. 21. N 14. P. 1578-1586. DOI:10.1002/cphc.202000296.

52. Yonekawa I., Mutoh K., Abe J. Visible light intensity dependent negative photochromism of binaphthyl-bridged phenoxyl-imidazolyl radical complex. Chem. Commun. 2019. V. 55. P. 1221-1224. DOI: 10.1039/c8cc09591d.

53. Yonekawa I., Mutoh, K., Kobayashi Y., Abe J. Intensity-dependent photoresponse of biphotochromic molecule composed of a negative and a positive photochromic unit. J. Amer. Chem. Soc. 2018. V. 140. N 3. P. 1091-1097. DOI: $10.1021 /$ jacs.7b11673.

54. Mutoh K., Miyashita N., Arai K., Abe J. Turn-On Mode Fluorescence switch by using negative photochromic imidazole dimer. J. Amer. Chem. Society. 2019. V. 141. P. 5650-5654. DOI: 10.1021/jacs.9b01870.

55. Jia S., Graham B., Capuano B., Tan A., Hawley A., Boyd B.J. Hexaarylbiimidazoles(HABI)-functionalized lyotropic liquid crystalline systems as visible light-responsive materials. J. Coll. Interf. Sci. 2020. V. 579. P. 379-390. DOI: 10.1016/j.jcis. 2020.06.006

56. Wang Q., Ligorio G., Schlesinger R., Diez- Cabanes V., Cornil D., Garmshausen Y., Hecht S., Cornil J., List-Kratochvil E.J. W., Koch N. Switching the electronic properties of $\mathrm{ZnO}$ surfaces with negative T- Type photochromic pyridyl- dihydropyrene layers and impact of Fermi level pinning. Adv. Mater. Interf. 2019. V. 6. N. 10. Art. 1900211. DOI: 10.1002/admi.201900211.

57. Klaue K., Garmshausen Y., Hecht S. Taking photochromism beyond visible: direct one-photon NIR photoswitches operating in the biological window. Angew. Chem. Intern. Ed. 2018. V. 57. N 5. P. 1414-1417. DOI: 10.1002/anie.201709554.

58. Sarkar R., Heitz M.-C., Roya G., Boggio-Pasqua M.J. Electronic excited states and UV-Vis absorption spectra of the dihydropyrene/cyclophanediene photochromic couple: a theoretical investigation. Phys. Chem. A. 2020. V. 124. P. 1567-1579. DOI: 10.1021/acs.jpca.9b11262.

59. Saima B., Khan N., Al-Faiyz Y.S., Ludwig R., Rehman W., Habib-ur-Rehman M., Sheikh N.S., Ayub K. Photo-tunable linear and nonlinear optical response of cyclophanediene-dihydropyrene photoswitches. J. Molec. Graph. Model. 2019. V. 88. P. 261-272. DOI: 10.1016/j.jmgm.2019.01.019.

60. Nemoto K., Enoki M., Katoh R., Suzuki K., Murase T., Imazeki S. Negative photochromism of a blue cyanine dye. Chem. Commun. 2020. V. 56. P. 15205-15207. DOI: 10.1039/d0cc06359b.

61. Hou I. C.-Y., Berger F., Narita A., Müllen K., Hecht S. Protongated ring- closure of a negative photochromic azulene- based diarylethene. Angew. Chem. Intern. Ed. 2020. V. 59. P. 18532 18536. DOI: 10.1002/anie.202007989.

62. Qian H., Pramanik S., Aprahamian I. Photochromic hydrazone switches with extremely long thermal half-lives. J. Amer. Chem. Soc. 2017. V. 139. N 27. P. 9140-9143. DOI: 10.1021/jacs.7b04993. 